\title{
Motoneurons Dedicated to Either Forward or Backward Locomotion in the Nematode Caenorhabditis elegans
}

\author{
Gal Haspel, ${ }^{1,2}$ Michael J. O'Donovan, ${ }^{1}$ and Anne C. Hart ${ }^{2}$ \\ ${ }^{1}$ Laboratory of Neural Control, Section on Developmental Neurobiology, National Institute of Neurological Disorders and Stroke, National Institutes of \\ Health, Bethesda, Maryland 20892, and 2Department of Neuroscience, Brown University, Providence, Rhode Island 02912
}

\begin{abstract}
Multifunctional motoneurons and muscles, which are active during forward and backward locomotion are ubiquitous in animal models. However, studies in the nematode Caenorhabditis elegans suggest that some locomotor motoneurons are necessary only for forward locomotion (dorsal B-motoneurons, DB), while others (dorsal A-motoneurons, DA) are necessary only for backward locomotion. We tested this hypothesis directly by recording the activity of these motoneurons during semirestrained locomotion. For this purpose, we used epifluorescence imaging of the genetically encoded calcium sensor cameleon, expressed in specific motoneurons, while monitoring locomotor behavior through the microscope condenser using a second camera. We found that ventral and dorsal B-motoneurons (DB and VB) were coactive during forward locomotion while ventral A-motoneurons (VA) were only active during backward locomotion. The signals we recorded correlated with the direction of locomotion but not with the faster undulatory cycles. To our knowledge, these are the first recordings of motoneuron activity in $C$. elegans and the only direction-dedicated motoneurons described to date.
\end{abstract}

\section{Introduction}

The neural basis of locomotor behavior in the nematode Caenorhabditis elegans is a particularly attractive system for study because of the simplicity of its neuromuscular system. C. elegans propels itself forward or backward by the sequential contraction of 95 muscle cells arranged in four quadrants along the body (Altun and Hall, 2009). In adult hermaphrodites, muscle cells of the body wall are innervated by 75 motoneurons comprising eight distinct classes (Fig. 1a) (White et al., 1986; Chen et al., 2006; Altun and Hall, 2009). Four classes innervate ventral muscle (VA, VB, VD, and VC) and four innervate dorsal muscle (DA, $\mathrm{DB}, \mathrm{DD}$, and AS). The involvement of specific classes of motoneurons in locomotion was explored in earlier neuronablation studies in young larvae when only three classes of locomotor motoneuron are present: DA, DB, and DD. Ablation of most DB motoneurons impairs forward but not backward locomotion. Conversely, ablation of most DA motoneurons impairs backward but not forward locomotion. Ablation of most DD motoneurons produces animals that are severely uncoordinated when moving in either direction (Chalfie et al., 1985). These experiments and others (Harbinder et al., 1997), along

\footnotetext{
Received May 2, 2010; revised June 23, 2010; accepted June 26, 2010.

This work was supported by funding from National Institutes of Health (NIH) National Institute of General Medical Sciences and National Institute of Neurological Disorders and Stroke (NINDS) (A.C.H.). G.H. was supported by The International Human Frontier Science Program Organization. The work was supported in part by the intramural program of the NIH NINDS. We thankS. Lockery and S. Faumont (University of Oregon) for their help and comments. YC2.60 and YC3.60 plasmids were a generous gift from A. Miyawaki (RIKEN, Japan). Some nematode strains used in this work were provided by the Caenorhabditis Genetics Center, which is funded by the NIH National Center for Research Resources.

Correspondence should be addressed to Anne C. Hart, Brown University, 60 Olive Street, SFH458 Mailbox GL-N, Providence, RI 02912. E-mail: Anne_Hart@brown.edu.

DOI:10.1523/JNEUROSCI.2244-10.2010

Copyright $\odot 2010$ the authors $\quad$ 0270-6474/10/3011151-06\$15.00/0
}

with neuronal morphology and connectivity (White et al., 1976), predicted that B-motoneurons (DB and VB) would be active during forward and A-motoneurons (DA and VA) during backward locomotion (Niebur and Erdös, 1993; Bryden and Cohen, 2008; Karbowski et al., 2008). Herein, we directly test this hypothesis by imaging motoneuron activity with a genetically encoded calcium sensor and correlating it to the direction of semirestrained locomotion.

\section{Materials and Methods}

Strains. C. elegans were cultivated at 15 or $25^{\circ} \mathrm{C}$ on NGM agar plates containing Escherichia coli (strain: OP50) according to standard techniques (Stiernagle, 2006). Transgenic wild type (N2 Bristol) animals expressing the calcium indicator yellow cameleon YC2.60 or YC3.60 (Nagai et al., 2004) in a subset of motoneuron classes were used in all studies. HA1722 (rtIs29 [acr-5p::YC2.60]) animals express cameleon in all VB and DB motoneurons and in some head and tail neurons; the latter were out of the field of view during calcium imaging. HA1706 and HA1708 (pha-1(e2123); rtEx630 or rtEx631 [pha-1(+); del$1 \mathrm{p}:$ :YC2.60]) express cameleon in all VB and VA motoneurons. TOL4 (aatIs4 [unc-17p::YC3.60]) express cameleon in all excitatory motoneurons.

Semirestrained preparation. The anterior portion of young adults or L4 larvae (between head and vulva) was restrained to a $1.5 \%$ agarose pad (saline as below, $\mathrm{pH}$ 9.0) with veterinary grade cyanoacrylate glue (Nexaband, Fisher). This stabilizes motoneurons within the glued region for imaging while the posterior body is free to move. This type of restraint has been used successfully to study leech (Baader and Kristan, 1992) and bullfrog tadpole (Rock et al., 1981) motor behaviors and assumes that the activity of neurons in the restrained region is similar to that in the moving part of the animal (see below for discussion). A coverslip with an agarose pad was placed on a cooling block $\left(0-5^{\circ} \mathrm{C}\right)$, to minimize animal movements during the gluing process. The coverslip with the glued animal was placed in a perfusion chamber (RC-21BDW, Warner Instruments) and covered with saline solution (modified M13 saline (100 mM NaCl, $10 \mathrm{~mm}$ 


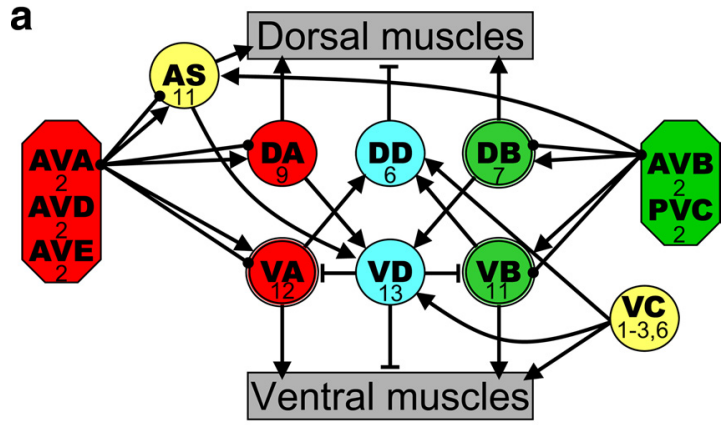

b

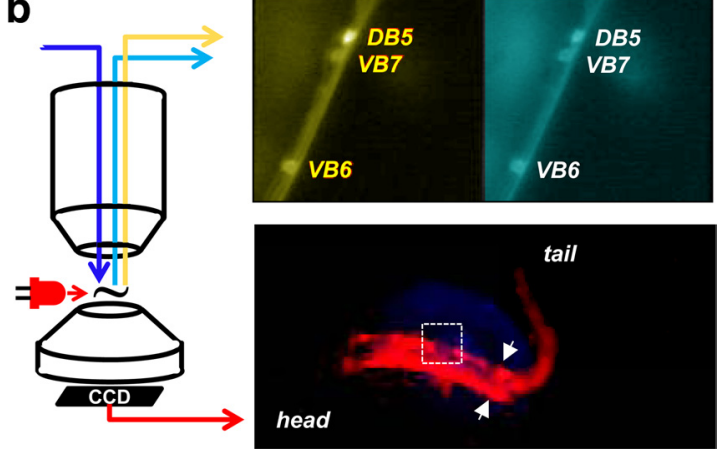

Figure 1. Locomotor neuronal circuit and recording set up. $\boldsymbol{a}$, Schematic of connectivity of the locomotor circuit [based on Chalfie and White (1988) and Von Stetina et al. (2006) with additional connections extracted from Altun and Hall (2009) and supplemental material of Chen et al. (2006)]: five pairs of interneurons (octagons) innervate eight groups of motoneurons (circles) that innervate either dorsal or ventral muscle of the body wall. Connecting lines represent excitatory (arrows) or inhibitory (T lines) synapses and gap junctions (solid circles). The number of neurons in each group is indicated. DB, VB, and VA, which were recorded from in this study, have a double-line frame. For simplicity, connectivity among interneurons is not presented and interneurons are grouped based on targets. $\boldsymbol{b}$, Recording set-up. Epifluorescence calcium imaging of motoneurons was done through a water-immersion objective on an upright microscope. An example of pseudocolored yellow and cyan channels is shown in the upper right panel. The nematode's behavior was imaged through the condenser using a modified webcam. The animal was illuminated with a red LED positioned close to the recording chamber. An image of the nematode viewed this way is shown in the lower right hand panel. The arrows indicate the caudal limit of the glued region and the field of view of the calcium imaging is shown by the white rectangle.

$\mathrm{KCl}, 10$ mм HEPES, 2 mм $\mathrm{MgCl}_{2}, 2$ mм CaCl ; pH $7.4(\mathrm{NaOH}) ; 350$ $\mathrm{mOsm}$ (glycerol)). Imaging sessions terminated at $30 \mathrm{~min}$ from tethering to avoid starvation (Sawin et al., 2000).

Calcium imaging. Two versions of the genetically encoded calcium indicator, Cameleon, were tested. Compared with Cameleon YC2.60, Cameleon YC3.60 has lower affinity for calcium (250 vs $40 \mathrm{~nm}$ ) (Nagai et al., 2004) and shorter time constant for decay ( 0.73 vs $5.24 \mathrm{~s}$ ) (Hendel et al., 2008). The signals acquired from YC3.60 were smaller and less reproducible than those acquired from YC2.60 ( $n=33$ paired recordings of VB and DB; and as observed before to a lesser extent by Hendel et al. (2008)) (see supplemental Fig. 1, available at www.jneurosci.org as supplemental material). Because switching between forward and backward locomotion is in the time scale of several seconds, the data presented were recorded with YC2.60.

Wide-field epifluorescence imaging was performed on a fixed stage upright microscope (Axioskop2FLplus) through a $63 \times, 0.95$ numerical aperture (NA) water-immersion Achroplan objective (Zeiss). For dualemission imaging of yellow cameleon an X-cite light source (EXFO), an excitation filter (D420/40X; all filters and mirrors from Chroma), a dichroic mirror (455dclp) and an image-splitter (W-view, Hamamatsu) were used. Two dichroic mirrors (500dcxr) in the image-splitter separate the emitted light into a cyan and a yellow image that were further filtered (470/30 and 535/30) and then projected side by side onto a cooled charge-coupled device (CCD) camera (Orca II ERG, Hamamatsu). Im- age stacks were acquired at $10 \mathrm{~Hz}$ using MetaVue software (Molecular Devices). A custom program (Jmalyze, written by R. Kerr and previously described (Kerr et al., 2000)) was used to track movement of individual motoneurons in the yellow channel and to measure the intensity of four regions of interest (ROI: cyan neuron and background and yellow neuron and background). IgorPro (ver. 5.03, WaveMetrics) and Matlab (ver. 7.4.0R2007a, MathWorks) were used for additional analyses. The average intensity of a background ROI of each channel was subtracted from the average intensity of the neuron ROI. The emission ratio was calculated as the ratio of yellow and cyan values and was divided by a fitted single exponent (correcting for photobleaching) to give $\Delta R / R$. The $\Delta R / R$ traces were denoised with a boxcar filter with a window of three time points before subsequent analysis. Signal-to-noise ratio (SNR) was calculated as the amplitude of the signal (filtered trace) divided by three times the root mean square of the noise (filtered trace subtracted from unfiltered). Traces with SNRs less than two were discarded. The mean SNR for traces included in the study was 5.2 (range: $2.0-15.8)$. Traces are presented in the figures without filtering. Signal sizes are presented as average $\Delta R / R$ as a percentage \pm SEM throughout text. Mean duration and interval for the imaging signals and for behavioral scoring were restricted to complete sequences within the analysis period. Incomplete sequences at the beginning and the end of the analysis period were discarded.

Behavior imaging. In some experiments, the behavior of the nematode was monitored and recorded during calcium imaging with a small CCD (extracted from a USB webcam) placed under the microscope condenser using the condenser as a low-magnification objective (Fig. 1b). A piece of filter-gel (\#27 Roscolux; Rosco) was positioned over the CCD and the nematode was illuminated by a red LED whose light was blocked by the filters used for the calcium imaging. The effective frame rate was $2.5 \mathrm{~Hz}$ ( $30 \mathrm{~Hz}$ refreshed every 12 frames). The webcam also recorded the excitation light used for calcium imaging, and this signal was used to synchronize the two sets of data. This set up is similar to that described by Faumont and Lockery (2006) which used an inverted microscope and an additional objective. Each behavior video was scored, blind to the calcium imaging data, at least twice for transitions between backward and forward semirestrained locomotion.

\section{Results}

VB and DB motoneurons are coactive, while VB and VA are never coactive during semirestrained locomotion

In an aqueous environment, C. elegans engage in nearly continuous, spontaneous locomotion when the anterior of their body is restrained. Neuronal activity in motoneuron cell bodies can be followed using the genetically encoded calcium indicator Yellow Cameleon that produces altered fluorescence ratio levels $(\Delta R / R)$ in response to changes in calcium levels (Nagai et al., 2004). To follow activity during semirestrained locomotion we used two different transgenic lines expressing YC2.60: either in VA and VB motoneurons or alternatively in VB and DB motoneurons. We found that neighboring ventral $\mathrm{A}$ and $\mathrm{B}$ motoneurons (VA, VB) exhibited antagonistic changes in activity during periods of semirestrained locomotion. Averaged peak values of the ratio changes were $16.8 \pm 2.4 \%$ (VA2-VA7, $n=20$ neurons) and $13.6 \pm 1.1 \%$ (VB3-VB7, $n=23$ neurons) respectively (Fig. $2 a$; 12 animals). In nematodes expressing the calcium sensor in ventral and dorsal $\mathrm{B}$-motoneurons (VB, DB), activity increased at the same time in both classes of motoneuron. Averaged peak values of the ratio changes were $14.5 \pm 1.0 \%$ (VB3-VB8, $n=35$ neurons) and $16.4 \pm 1.6 \%$ (DB3-DB5, $n=18$ neurons), respectively (Fig. $2 b$; 17 animals).

The mean duration and interval between these signals were $12.0 \pm 0.7 \mathrm{~s}$ and $6.7 \pm 0.4 \mathrm{~s}$, respectively, corresponding to the duration and interval between forward and backward movements, but were considerably slower than the undulation fre- 
a
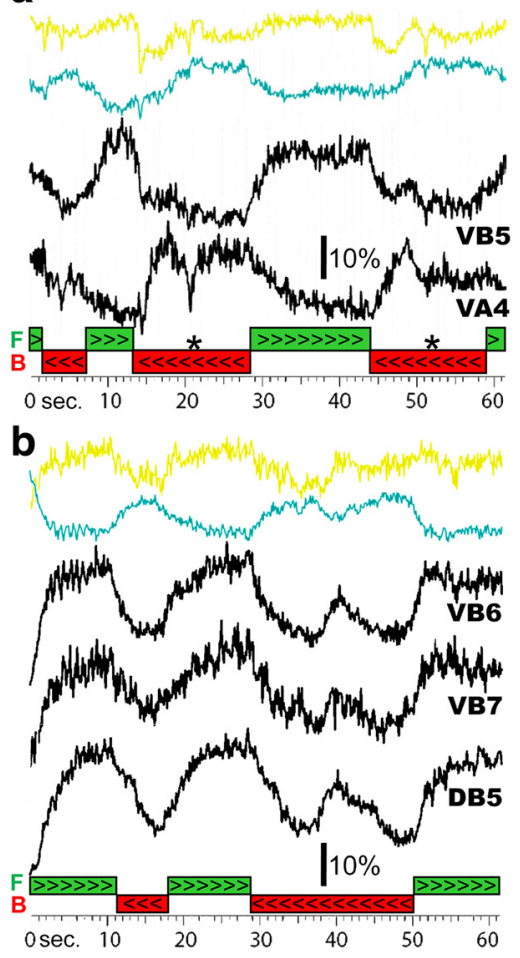

C

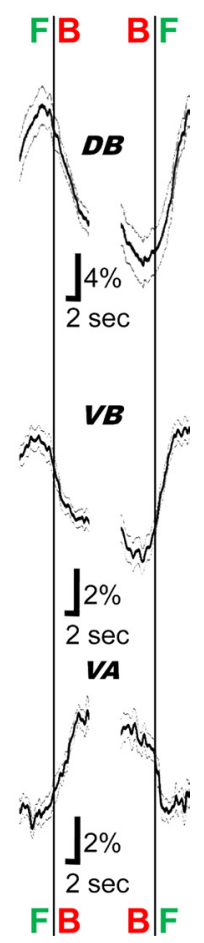

Figure 2. B-motoneurons are active during forward semirestrained locomotion while A-motoneurons are active during backward. $\boldsymbol{a}$, The top two colored traces are the signals from the yellow and cyan channels recorded from a VB5 motoneuron. The ratio of the yellow/cyan signal is shown underneath these traces together with the ratio signal from a VA4 motoneuron during forward (green bar $\gg$ ) and backward (red bar «) locomotion. The asterisk $\left({ }^{*}\right)$ marks a period when locomotion stopped. $\boldsymbol{b}$, Dorsal and ventral B-motoneurons, The raw yellow and cyan traces and their ratio recorded from a VB6 motoneuron (yellow and cyan and uppermost black traces) together with the activity of a VB7 motoneuron (middle black trace) and a DB5 motoneuron (bottom black trace) during forward (green bar $)$ ) and backward (red bar «) locomotion. c, Mean ratio change during the switch from forward to backward (left traces) and backward to forward (right traces) locomotion. All ratiometric recordings were aligned by the time of the independently scored changes in direction determined from the behavior video. The number of transitions used to compute the averages were as follows: forward to backward, 12 (DB), 60 (VB), 23 (VA); backward to forward, 13 (DB), 55 (VB), 18 (VA). Gray traces are one SEM.

quency $(0.74 \mathrm{~Hz} \pm 0.03)$. In the next section, we compare the fluorescence changes in motoneurons with the behaviorally recorded forward and backward sequences and quantify the temporal relationships between the behavior and the activity of the A and B motoneurons.

\section{VB and DB motoneurons are active during forward and VA during backward locomotion}

In 14 animals, we monitored locomotor behavior at the same time as the calcium imaging. This allowed us to relate the fluorescence changes in specific motoneurons to the direction of movement (see Materials and Methods and Fig. 1b). As described previously (Faumont et al., 2005), two locomotor states occur during semirestrained locomotion: a fast and shallow dorsoventral bending $(0.74 \pm 0.03 \mathrm{~Hz}$, range: $0.38-1.25 \mathrm{~Hz})$ associated with forward locomotion and a slower, deeper bending $(0.20 \pm$ $0.01 \mathrm{~Hz}$, range: $0.08-0.44 \mathrm{~Hz}$ ) associated with backward locomotion. Each bout of directional locomotion consisted of several dorsoventral undulations. Some bouts were interrupted by a period in which the animal would remain bent either dorsally or ventrally for a few seconds (e.g., Fig. $2 a$ ). These stops could cor-

respond to slow backward locomotion, Omega-turns (Faumont et al., 2005), or rest (Wakabayashi et al., 2004) and were excluded from this study. We found that B-motoneurons were active during forward locomotion, while A-motoneurons were active during backward locomotion.

We scored the behavior movies for changes in the direction of semirestrained locomotion and used the times when a change of direction occurred to select sections of the calcium imaging data ( $5 \mathrm{~s}$ before and $5 \mathrm{~s}$ after) for further analysis. This was done blind to the calcium imaging data.

Averaged responses (Fig. 2c) demonstrate that the decrease in calcium levels in $\mathrm{DB}(n=12$ changes in direction) and VB motoneurons $(n=60)$ coincided with an increase in VA motoneurons $(n=23)$, and these changes preceded a switch from backward to forward locomotion. A corresponding increase in calcium levels in $\mathrm{DB}(n=13)$ and VB motoneurons $(n=55)$ coincided with a decrease in VA motoneurons $(n=18)$ and preceded a switch from backward to forward locomotion. The change in direction of locomotion did not require the calcium level to reach maximal levels (Fig. $2 c$; e.g., Fig. $2 a, b$ ). However, the slow time constant of YC2.60 might affect the shape of the calcium signals (Hendel et al., 2008) as we discuss below.

$\mathrm{B}$-motoneurons of the same class are connected by gap junctions and could sequentially activate each other along the ventral cord to signal the change in direction. However, when we examined two adjacent VAs or two VBs (e.g., VB6 and VB7 in Fig. 2b), there was no apparent delay in the onset of the calcium rise at the beginning of a bout of locomotion in 14 out of 18 animals; the remaining four cases, either the anterior (three) or posterior (one) VB motoneuron was relatively delayed during both backward and forward locomotion.

\section{No dorsoventral alternating activity was recorded in B-motoneurons}

Dorsal and ventral B-motoneurons innervate antagonistic muscles and were hypothesized to exhibit alternating activity correlated with the body undulations during forward locomotion (Niebur and Erdös, 1993; Bryden and Cohen, 2008; Karbowski et al., 2008). Both classes of motoneuron possess long undifferentiated posterior processes that may function as a stretch receptor (White et al., 1986). The frequency of sinusoidal movements during semirestrained forward locomotion was $0.74 \pm 0.03 \mathrm{~Hz}$, which is slower than reported for free locomotion $(1.46 \mathrm{~Hz}$; Tsechpenakis et al. (2008), 0.8/2.1 Hz crawl/swim; PierceShimomura et al. (2008)). We did not observe cameleon ratio changes that correlated with undulatory rhythms. To maximize the detection of signal at these undulation frequencies, we filtered $(0.25-4 \mathrm{~Hz})$ the ratiometric signal recorded in a dorsal $\mathrm{B}$-motoneurons and their ventral counterparts (namely DB3/ VB3, DB4/VB5, and DB5/VB7), during bouts of forward locomotion before cross-correlation analysis (Fig. $3 a$ ). The processes of DB4, VB5, DB5, and VB7 extend beyond the vulva (D. Hall, personal communication) and are therefore subject to the movements of the unrestrained posterior part of the body. As a result, their putative sensory feedback should be similar to that occurring in free locomotion. If ventral and dorsal neurons alternated their activity, we expected an anti-phase (negative) cross-correlation of neuronal activity. Instead, only 5 of 32 pairs of recordings exhibited any cross-correlation of activity in the $0.25-4 \mathrm{~Hz}$ frequency range and those were weakly in-phase (Fig. $3 b$ ). Moreover, the amplitude of these synchronous signals was much smaller than the signal associated with the direction of locomotion discussed above. In addition, we cannot exclude the possi- 

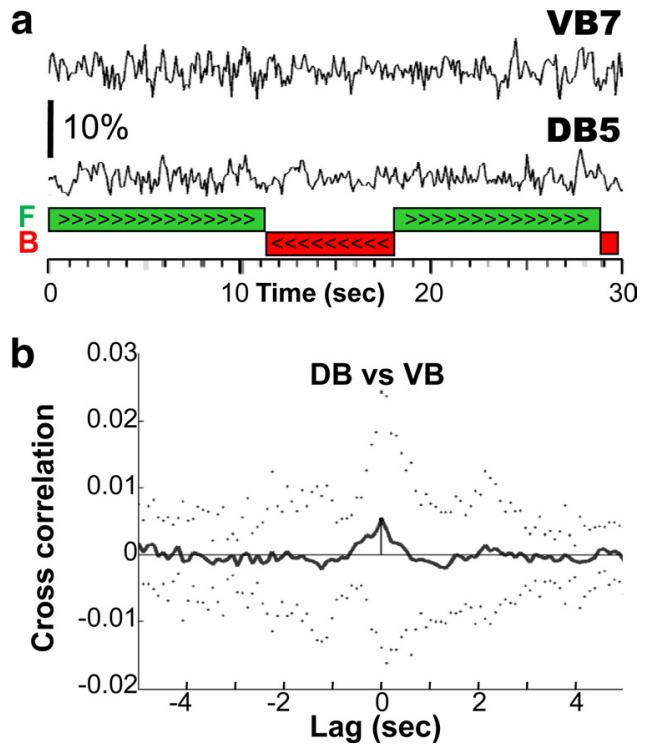

Figure 3. No alternating activity associated with the body undulation was recorded in neighboring VB and DB motoneurons. $\boldsymbol{a}$, The traces are the first $30 \mathrm{~s}$ of the bandpass filtered $(0.25-4 \mathrm{~Hz})$ middle (VB7) and bottom (DB5) ratio traces shown in Figure $2 b$. No alternating activity between VB7 and DB5 is evident during the two bouts of forward locomotion (green bars), which comprised 8 and 11 undulations, respectively. $\boldsymbol{b}$, Mean (solid line) and SD (dotted line) cross-correlation of ratio changes in all adjacent pairs of VB and DB motoneurons ( $n=32 \mathrm{DB} / \mathrm{VB}$ pairs). Ratio traces were bandpass filtered $(0.25-4 \mathrm{~Hz})$ before cross-correlation.

bility that these small signals are artifacts, resulting from uncompensated movement of the motoneurons in the imaged field. Nonetheless, in none of our recordings could we detect alternating activity between the dorsal and ventral motoneurons ( $n=32$ pairs). Therefore, given the limitations of calcium imaging (see below), our results do not support or contradict models in which ventral and dorsal B-motoneurons alternate in activity during forward locomotion.

\section{Discussion}

We found that tonic fluorescence changes in the B-motoneurons (VB and $\mathrm{DB}$ ) and A-motoneurons (VA) were mutually exclusive and correlated with the direction of locomotion. B-motoneurons were coactive during forward locomotion, while A-motoneurons were active during backward locomotion. To our knowledge, this is the first demonstration of a specialized function for motoneurons in forward and backward locomotion. Furthermore, it is the first recording of motoneuron activity driving locomotion in $C$. elegans.

Our results demonstrate that $\mathrm{DB}, \mathrm{VB}$, and VA classes of motoneurons are active during one direction of locomotion but not the other, consistent with earlier lesion studies showing that DA and DB were necessary for backward or forward locomotion respectively (Chalfie et al., 1985). The other classes of motoneurons have not yet been recorded from. This suggests that at least 39 ( $7 \mathrm{DB}, 11 \mathrm{VB}, 12 \mathrm{VA}$, and $9 \mathrm{DA})$ of the 75 motoneurons that innervate locomotor muscles are dedicated to one direction of locomotion or another. In contrast to motoneurons, locomotor muscles are multifunctional and are active during both directions of locomotion (PierceShimomura et al., 2008). This is because each muscle cell is innervated by four to eight motoneurons of different classes (White et al., 1976; Chen et al., 2006).

\section{Failure to detect sinusoidal alternating activity between VB} and DB motoneurons

Existing models for C. elegans locomotion (Niebur and Erdös, 1993; Bryden and Cohen, 2008; Karbowski et al., 2008) address only forward locomotion during crawling. In all of these models, $\mathrm{VB}$ and $\mathrm{DB}$ are the sources of alternating activity to ventral and dorsal muscle. We were not able to discern any such alternating activity between pairs of VB and DB motoneurons that innervate contralateral muscle cells. If alternating motoneuron activity does occur, then one of several reasons may account for our failure to detect it. First, the activity of motoneurons at the recording site might be affected by the tethering. In addition, the decay time constant of YC2.60 we used to detect calcium changes accompanying forward and backward locomotion was too slow (5.2 s) (Hendel et al., 2008) to allow us to resolve the faster oscillatory signals. Although YC3.60 has a faster decay time constant, the signals it generated were consistently smaller than YC2.60 and fast rhythmic signals accompanying sinusoidal movements could not be detected (supplemental Fig. 1, available at www.jneurosci. org as supplemental material). Finally, we cannot exclude the possibility that changes in calcium levels recorded in the cell body are decoupled from the activity of neuronal processes and the neuromuscular junction. The relationship between motoneuronal calcium levels and membrane potential is not known in $C$. elegans. As a result, calcium signals may not reflect changes in membrane potential. This is consistent with earlier work indicating that genetically encoded calcium indicators cannot monitor fast, subthreshold or hyperpolarizing activity (Jayaraman and Laurent, 2007; Hendel et al., 2008).

The movements generated during semirestrained locomotion are not identical to those occurring in free locomotion. For example, during semirestrained locomotion, backward cycles are larger in amplitude and longer than those of free locomotion. At present it is not possible to image motoneuron activity during free locomotion, although recent technical developments may allow this goal to be accomplished (Chronis et al., 2007; Ben Arous et al., 2010). Despite the differences between semirestrained and free locomotion, work in other systems suggests that it is unlikely that the partial restraint used in these experiments results in a completely novel mode of operation of the motoneuronal network.

The results presented here cannot establish the presence or absence of alternating activity. It is possible, for example, that A and $\mathrm{B}$ classes of motoneurons remain tonically active during backward and forward locomotion, respectively, and do not contribute to sinusoidal oscillations. Other cells may be the source of alternating activity. For example, alternating activity could be carved out of tonically activated muscle by phasic activity of inhibitory D class motoneurons (VD and DD). Consistent with this idea, laser ablation of DD motoneurons prevented both forward and backward locomotion in newly hatched larvae (Chalfie et al., 1985). Rhythmic activity has been described in Ascaris suum inhibitory motoneurons (Angstadt and Stretton, 1989; Davis and Stretton, 1989) but was not found to correlate with body undulation in a semi-intact preparation (Stretton et al., 1992). However, adult mutants that lack functional GABAergic transmission (e.g., unc-25/GAD mutants (McIntire et al., 1993)) and nematodes in which GABAergic transmission was genetically blocked in the adult (Davis et al., 2008) do exhibit both forward and backward sinusoidal locomotion, which is at odds with this hypothesis. Alternatively, muscle cells may produce the oscillations, while the motoneurons modulate their amplitude. This model was suggested by White et al. (1976) but lacks experimental sup- 
port. Rhythmic activity of muscle cells is not predicted by an electrophysiological model (Boyle and Cohen, 2008) and has not been reported in dissected preparations (Richmond and Jorgensen, 1999), nor in primary cultures (Christensen et al., 2002). Last, an alternative source of rhythmic activity might be excitatory motoneurons classes AS, VC or both, but their role in C. elegans locomotion has not been explored.

\section{Evolutionary considerations and comparison with other species}

Backward, as well as forward, locomotion is exhibited by many animal species. However, only a few studies have addressed the mechanism of backward locomotion directly. In cat, human, and lamprey, it has been suggested that common spinal central pattern generators are used for forward and backward locomotion (Buford and Smith, 1990; Matsushima and Grillner, 1992; Lamb and Yang, 2000; Zehr and Hundza, 2005). In the stick insect, backward walking appears to be produced not by reversing the forward locomotion pattern but by overriding the forward pattern (Graham and Epstein, 1985). In all of these preparations, the motoneurons are multifunctional and are active during both forward and backward locomotion.

By contrast, it appears that specific motoneurons are dedicated to a particular direction of movement in the nematode. Interneurons in the nematode appear to release one pattern or another rather than participate directly in pattern generation, as in the other animals described. This is supported by tonic activation of AVA interneurons during backward locomotion (Chronis et al., 2007; S. Faumont, T. R. Thiele, and S. R. Lockery, personal communication) and by the common innervation of A-motoneurons by AVA, AVD, and AVE (anterior only) interneurons and of B-motoneurons by AVB and PVC interneurons. Furthermore, the synchronous onset of calcium transients in neighboring VB or neighboring VA motoneurons at the beginning of locomotion suggests a common source of activation for motoneurons of the same class along the ventral cord. While the relatively slow decay time constant of YC2.60 might interfere with resolving sequential activity during a bout locomotion, it should not affect the onset of the signal at the onset of a bout.

A neuronal network comprised of direction-specific classes of motoneurons might be an ancestral form of locomotor control to which dedicated and multifunctional interneurons were subsequently added and that then assumed the role of pattern generation. This would have freed motoneurons to become multifunctional, thereby greatly extending the repertoire of motor behaviors. In the nematode, a motoneuronal pattern-generating mechanism might suffice because the behavioral repertoire is limited, and the total number of motoneurons and muscle cells is relatively small.

\section{References}

Altun ZF, Hall DH (2009) Nervous system, general description. In: WormAtlas. doi:10.3908/wormatlas.1.18.

Angstadt JD, Stretton AO (1989) Slow active potentials in ventral inhibitory motor neurons of the nematode Ascaris. J Comp Physiol A 166:165-177.

Baader AP, Kristan WB Jr (1992) Monitoring neuronal activity during discrete behaviors: a crawling, swimming and shortening device for tethered leeches. J Neurosci Methods 43:215-223.

Ben Arous J, Tanizawa Y, Rabinowitch I, Chatenay D, Schafer WR (2010) Automated imaging of neuronal activity in freely behaving Caenorhabditis elegans. J Neurosci Methods 187:229-234.

Boyle JH, Cohen N (2008) Caenorhabditis elegans body wall muscles are simple actuators. Biosystems 94:170-181.
Bryden J, Cohen N (2008) Neural control of Caenorhabditis elegans forward locomotion: the role of sensory feedback. Biol Cybern 98:339-351.

Buford JA, Smith JL (1990) Adaptive control for backward quadrupedal walking. II. Hindlimb muscle synergies. J Neurophysiol 64:756-766.

Chalfie M, White JG (1988) The nervous system. In: The nematode Caenorhabditis elegans (Wood WB, ed), pp 337-391. Cold Spring Harbor, NY: Cold Spring Harbor Laboratory.

Chalfie M, Sulston JE, White JG, Southgate E, Thomson JN, Brenner S (1985) The neural circuit for touch sensitivity in Caenorhabditis elegans. J Neurosci 5:956-964.

Chen BL, Hall DH, Chklovskii DB (2006) Wiring optimization can relate neuronal structure and function. Proc Natl Acad Sci U S A 103:47234728 .

Christensen M, Estevez A, Yin X, Fox R, Morrison R, McDonnell M, Gleason C, Miller DM 3rd, Strange K (2002) A primary culture system for functional analysis of $C$. elegans neurons and muscle cells. Neuron 33:503-514.

Chronis N, Zimmer M, Bargmann CI (2007) Microfluidics for in vivo imaging of neuronal and behavioral activity in Caenorhabditis elegans. Nat Methods 4:727-731.

Davis MW, Morton JJ, Carroll D, Jorgensen EM (2008) Gene activation using FLP recombinase in C. elegans. PLoS Genet 4:e1000028.

Davis RE, Stretton AO (1989) Signaling properties of Ascaris motorneurons: graded active responses, graded synaptic transmission, and tonic transmitter release. J Neurosci 9:415-425.

Faumont S, Lockery SR (2006) The awake behaving worm: simultaneous imaging of neuronal activity and behavior in intact animals at millimeter scale. J Neurophysiol 95:1976-1981.

Faumont S, Miller AC, Lockery SR (2005) Chemosensory behavior of semirestrained Caenorhabditis elegans. J Neurobiol 65:171-178.

Graham D, Epstein S (1985) Behaviour and motor output for an insect walking on a slippery surface: II. Backward walking. J Exp Biol 118:287-296.

Harbinder S, Tavernarakis N, Herndon LA, Kinnell M, Xu SQ, Fire A, Driscoll M (1997) Genetically targeted cell disruption in Caenorhabditis elegans. Proc Natl Acad Sci U S A 94:13128-13133.

Hendel T, Mank M, Schnell B, Griesbeck O, Borst A, Reiff DF (2008) Fluorescence changes of genetic calcium indicators and OGB-1 correlated with neural activity and calcium in vivo and in vitro. J Neurosci 28:7399-7411.

Jayaraman V, Laurent G (2007) Evaluating a genetically encoded optical sensor of neural activity using electrophysiology in intact adult fruit flies. Front Neural Circuits 1:3.

Karbowski J, Schindelman G, Cronin CJ, Seah A, Sternberg PW (2008) Systems level circuit model of C. elegans undulatory locomotion: mathematical modeling and molecular genetics. J Comput Neurosci 24:253-276.

Kerr R, Lev-Ram V, Baird G, Vincent P, Tsien RY, Schafer WR (2000) Optical imaging of calcium transients in neurons and pharyngeal muscle of C. elegans. Neuron 26:583-594.

Lamb T, Yang JF (2000) Could different directions of infant stepping be controlled by the same locomotor central pattern generator? J Neurophysiol 83:2814-2824.

Matsushima T, Grillner S (1992) Neural mechanisms of intersegmental coordination in lamprey: local excitability changes modify the phase coupling along the spinal cord. J Neurophysiol 67:373-388.

McIntire SL, Jorgensen E, Horvitz HR (1993) Genes required for GABA function in Caenorhabditis elegans. Nature 364:334-337.

Nagai T, Yamada S, Tominaga T, Ichikawa M, Miyawaki A (2004) Expanded dynamic range of fluorescent indicators for $\mathrm{Ca}(2+)$ by circularly permuted yellow fluorescent proteins. Proc Natl Acad Sci USA 101:10554-10559.

Niebur E, Erdös P (1993) Theory of the locomotion of nematodes: control of the somatic motor neurons by interneurons. Math Biosci 118:51-82.

Pierce-Shimomura JT, Chen BL, Mun JJ, Ho R, Sarkis R, McIntire SL (2008) Genetic analysis of crawling and swimming locomotory patterns in C. elegans. Proc Natl Acad Sci U S A 105:20982-20987.

Richmond JE, Jorgensen EM (1999) One GABA and two acetylcholine receptors function at the C. elegans neuromuscular junction. Nat Neurosci 2:791-797.

Rock MK, Hackett JT, Brown DL (1981) Does the Mauthner cell conform to the criteria of the command neuron concept? Brain Res 204:21-27. 
Sawin ER, Ranganathan R, Horvitz HR (2000) C. elegans locomotory rate is modulated by the environment through a dopaminergic pathway and by experience through a serotonergic pathway. Neuron 26:619-631.

Stiernagle T (2006) Maintenance of C. elegans. In: WormBook (The C. elegans Research Community, WormBook, ed), doi/10.1895/wormbook.1.101.1. http://www.wormbook.org.

Stretton A, Donmoyer J, Davis R, Meade J, Cowden C, Sithigorngul P (1992) Motor behavior and motor nervous system function in the nematode Ascaris suum. J Parasitol 78:206-214.

Tsechpenakis G, Bianchi L, Metaxas D, Driscoll M (2008) A novel computational approach for simultaneous tracking and feature extraction of $C$. elegans populations in fluid environments. IEEE Trans Biomed Eng 55:1539-1549.
Von Stetina SE, Treinin M, Miller DM 3rd (2006) The motor circuit. Int Rev Neurobiol 69:125-167.

Wakabayashi T, Kitagawa I, Shingai R (2004) Neurons regulating the duration of forward locomotion in Caenorhabditis elegans. Neurosci Res 50:103-111.

White JG, Southgate E, Thomson JN, Brenner S (1976) The structure of the ventral nerve cord of Caenorhabditis elegans. Philos Trans R Soc Lond B Biol Sci 275:327-348.

White JG, Southgate E, Thomson JN, Brenner S (1986) The structure of the nervous system of the nematode C. elegans. Philos Trans R Soc Lond B Biol Sci 314:1-340.

Zehr EP, Hundza SR (2005) Forward and backward arm cycling are regulated by equivalent neural mechanisms. J Neurophysiol 93:633-640. 